\title{
V1: Stationäre Krankenpflege im Blickpunkt einer langfristigen Mitarbeiterbindung - eine Status-quo-Analyse
}

\author{
Sabine Löffert
}

Online publiziert: 30. Oktober 2013

(C) Springer-Verlag Wien 2013

Einleitung: Der demografische Wandel betrifft nicht nur Patienten sondern auch das Krankenhauspersonal, welches sich, insbesondere im Pflegebereich, zukünftig dem Problem einer zunehmenden Alterung gegenübersieht. Vor dem Hintergrund sich verringernder Nachwuchskräfte und des bestehenden Fachkräftemangels ist es für die Krankenhäuser daher umso wichtiger, Mitarbeiter verschiedener Altersgruppen durch eine zielgruppenorientierte Gestaltung der Arbeitsplätze langfristig an das Krankenhaus zu binden und das Krankenhaus altersübergreifend zu einem attraktiven Arbeitsplatz zu machen.

Methodik: In einer repräsentativen Umfrage unter den Pflegedienstleitungen der Allgemeinkrankenhäuser in Deutschland wurde eine Bestandaufnahme der Situation erstellt. Insgesamt beteiligten sich $n=309$ Krankenhäuser an der Studie. Alternsgerechtes Arbeiten wurde dabei explizit nicht nur als Anpassung der Arbeitsplätze und der Arbeitsbedingungen an die Bedürfnisse älterer Arbeitnehmer betrachtet, sondern zieht auch auf verschiedene Altersgruppen ausgerichtete Arbeitsbedingungen in Betracht. Dazu wurde speziell ein standardisierter Fragebogen „Alter(n)sgerechtes Arbeiten im Krankenhaus" entwickelt.
Ergebnisse: Die Ergebnisse der Umfrage zeigten deutlich, dass das Problem einer zunehmenden Alterung der Pflegekräfte in den Kliniken erkannt wurde und bereits gehandelt wird. In vielen Einrichtungen sind daher Ansätze in der Personalplanung zum Ausgleich der Altersstruktur der Mitarbeiter gut sichtbar. Möglichkeiten zur alternsgerechten Arbeitsplatzgestaltung werden häufig genutzt, Gesundheitsfördermaßnahmen für Pflegemitarbeiter weitreichend geboten. Arbeitsorganisatorische Maßnahmen speziell für ältere Mitarbeiter werden dagegen nur punktuell umgesetzt.

Schlussfolgerungen: Aufgrund der häufig herrschenden limitierten personellen und finanziellen Ressourcen können in den Krankenhäusern oft nur wenig spezielle Maßnahmen für (ältere) Mitarbeiter in der Pflege umgesetzt werden. Es fehlt nicht selten an praxistauglichen Konzepten und Strategien. Die Kliniken arbeiten jedoch aktiv, je nach Möglichkeiten, an der Bindung ihrer Mitarbeiter im Pflegebereich.

S. Löffert $(\square)$

Deutsches Krankenhausinstitut e. V.,

Geschäftsbereich Forschung, Düsseldorf, Deutschland

E-Mail: sabine.loeffert@dki.de 